Revue d'histoire de l'enfance « irrégulière »

Le Temps de l'histoire

$11 \mid 2009$

Paroles libres, paroles captives

\title{
Ludivine Bantigny, Ivan Jablonka (dir.), Jeunesse oblige.
}

Anne-Claire Rebreyend

\section{(2) OpenEdition}

1 Journals

Édition électronique

URL : http://journals.openedition.org/rhei/3102

DOI : 10.4000/rhei.3102

ISBN : 978-2-7535-1650-2

ISSN : 1777-540X

Éditeur

Presses universitaires de Rennes

Édition imprimée

Date de publication : 1 octobre 2009

ISBN : 978-2-7535-0927-6

ISSN : 1287-2431

Référence électronique

Anne-Claire Rebreyend, « Ludivine Bantigny, Ivan Jablonka (dir.), Jeunesse oblige. », Revue d'histoire de l'enfance « irrégulière » [En ligne], 11 | 2009, mis en ligne le 01 octobre 2009, consulté le 04 décembre 2020. URL : http://journals.openedition.org/rhei/3102 ; DOI : https://doi.org/10.4000/rhei.3102

Ce document a été généré automatiquement le 4 décembre 2020

(C) PUR 


\title{
Ludivine Bantigny, Ivan Jablonka (dir.), Jeunesse oblige.
}

\author{
Anne-Claire Rebreyend
}

\section{Jeunesse oblige. Histoire des jeunes en France XIX ${ }^{e}-X X I^{e}$ siècle.}

2 Ludivine Bantigny et Ivan Jablonka (dir.)

3 Paris, PUF, 2009, 307 p. (préface de Jean-François Sirinelli).

4 ISBN : 978-2-13-056692-2

5 Dirigé par Ludivine Bantigny et Ivan Jablonka, Jeunesse oblige rassemble dix-huit contributions sur le thème de la jeunesse selon un découpage chronologique: naissance de la jeunesse contemporaine (XIXe siècle-début du XXe); de la jeunesse aux jeunes (d'un aprèsguerre à l'autre); vers une identité juvénile ? (des années 1960 à nos jours).

6 Dans une lumineuse introduction, Ludivine Bantigny rappelle le riche héritage historiographique dans lequel s'inscrit l'ouvrage, tout en mettant l'accent sur les nouvelles problématiques qu'il propose. Elle cite d'abord Pierre Bourdieu ( «la jeunesse n'est qu'un mot») pour souligner la difficulté à circonscrire ce que sont «les jeunes», et «la jeunesse » (p. 6). Relevant ensuite l'extension de la notion de jeunesse aux XIXe et XXe siècles et sa plus grande visibilité au cours des trente dernières années (pp. 9-10), l'historienne insiste sur la finalité sociale et la fonction politique de la jeunesse d'où l'importance d'en faire l'histoire (p. 11).

7 Jeunesse oblige propose effectivement une histoire des jeunes en France en multipliant les angles d'approche. Le rapport entre les jeunes et la politique est ainsi interrogé à travers différentes époques. Pour Jean-Claude Caron, la jeunesse se réduit entre 1815 et 1870 au « jeune homme » d'origine sociale élevée ayant « une mission d'action, d'héroïsme, et, ce faisant, une mission politique » (p. 21). C'est lui qui 
est mis en avant sur les barricades de 1830 ou de 1848, comme si les révolutions en France se résumaient à des conflits de générations. S'intéressant plus spécifiquement aux étudiants, à travers la littérature du XIXe siècle, Pierre Moulinier montre qu'ils sont à la fois critiqués et encouragés à donner l'exemple (par Michelet ou par Lavisse) et prennent conscience de former un groupe influent, susceptible de devenir une force politique et syndicale (p. 30). En revanche, la jeunesse rurale intéresse moins les élites et joue un maigre rôle dans les luttes sociales du XIXe siècle. Jean-Claude Farcy cherche à comprendre, à l'aide de monographies (Le Play) et de recensements, ce « silence des prolétaires de la terre », alors qu'ils sont si nombreux (p. 51). Le poids considérable des jeunes dans le monde du travail rural, le fait que cette jeune main-d'œuvre espère échapper rapidement à sa condition, ne conduit pas à la révolte. Plus proche de nous, la difficile mobilisation des jeunes Maghrébins est relatée par Richard Derderian. Dès la fin des années 1970, et surtout dans les années 1980, les jeunes Maghrébins ont lutté pour la reconnaissance ; la Marche pour l'égalité et contre le racisme de 1981 constituant le point culminant du « mouvement beur» (p. 269). Mais les émeutes urbaines de 2005 et de 2007 n'ont pas suscité d'organisations offrant des solutions constructives car les jeunes Maghrébins souffrent d'un déficit de « mémoire collective » (p. 276).

8 L'ouvrage fait également le point sur les liens entre les jeunes et la culture et sur la réalité d'une «culture jeune ». Rappelant le succès du cinéma de la Nouvelle Vague dans les années 1950, Antoine de Baecque critique l'idée d'un cinéma de droite : le cinéma de la Nouvelle Vague n'est pas politique par son message, mais par sa manière de filmer la jeunesse, de capter ses habitudes à tel point qu'une génération de jeunes Français (appelée Nouvelle Vague dans la presse) s'y retrouve (p. 178). La période 1962-1968, en revanche, est moins riche sur le plan de la rencontre entre jeunesse et cinéma, indique Sébastien Le Pajolec dans une étude sur les rapports entre cinéma et "yéyés ». Il souligne l'absence de cinéma yéyé en France à l'exception du film D'où viens-tu Johnny ? (p. 192). Les scopitones (films courts passés dans les cafés) et les photographies de Salut les copains ont plus servi les artistes yéyés que le cinéma. De fait, c'est surtout le rock'n'roll qui entraîne, entre 1956 et 1966, la construction d'une subculture adolescente, dont Florence Tamagne décrit les nuances. Considéré comme une «musique de jeunes », le rock doit être fait par et pour des jeunes (p. 203), même si les amateurs de rock sont loin de former un groupe unitaire, asexué et international. Puis, dans le milieu des années 1960, l'intellectualisation et la politisation du discours sur le rock constitue un « passage à l'âge adulte » de cette musique (p. 212).

Les évolutions du service militaire au fil des XIXe et XXe siècles permettent d'interroger la construction d'une identité juvénile masculine. Pour Annie Crépin et Odile Roynette, la création du service militaire obligatoire durant la Révolution française fait de l'armée un lieu de socialisation de la jeunesse, fortement contesté durant la 
période révolutionnaire et impériale. À partir du Second Empire, l'insoumission diminue et la lente acceptation du service militaire, identifié comme un passage obligé pour «devenir un homme», se poursuit durant la Troisième République (p. 76). Il s'agit alors d'une expérience valorisante, constitutive de l'identité masculine (p. 82). Cette idée perdure chez les jeunes Français au cours des années 1960-1970 (surtout chez les ouvriers et les paysans, moins chez les étudiants) affirme Christian Gracieux (p. 217). Avec la fin du conseil de révision en 1970 supplanté par une sélection de trois jours, l'allongement de la scolarité et la suppression des sursis d'incorporation, la baisse de l'âge de la majorité électorale et civile, le service militaire perd sa fonction traditionnelle de rite de passage de la jeunesse à l'âge adulte et «d'école des hommes » pour devenir un simple « impôt temporel » (p. 224).

10 Si l'image du militaire s'impose dans les esprits, la figure du jeune délinquant - autre figure essentiellement masculine - reste floue durant tout le XIXe siècle rappelle Jean-Jacques Yvorel. Durant ce siècle, la délinquance juvénile commence à être dissociée de la délinquance en général, mais les enquêtes sociales et les discours scientifiques ne font que reprendre des topoï de la littérature ou des médias sur la figure du jeune délinquant sans réaliser de véritables enquêtes de terrain ni d'études de cas concrets (p. 94). On ne sait donc pas grand chose des délinquants en chair et en os, mais ceux-ci sont regroupés dans une catégorie pénale spécifique à partir de la loi de 1906 : celle des 16-18 ans. Pascale Quincy-Lefebvre relate la genèse de la loi et les débats sur la question du «discernement » au moment des faits : si le délinquant est «non discernant», il est protégé par la loi et doit être éduqué. Au contraire, s'il est capable de « distinguer le bien du ma »l, le délinquant doit être condamné comme un majeur (il n'y a pas d'excuse de la minorité avant 1945). La justice peut alors contrôler une population jugée dangereuse (p. 101). Sarah Fishman poursuit l'analyse de la délinquance juvénile sous le régime de Vichy. Le passage d'un système judiciaire axé sur le châtiment à un système basé sur l'action éducative s'y poursuit avec la loi du 27 juillet 1942 qui met fin à la notion de « discernement». Malgré l'intention progressiste de la réforme, la nature autoritaire du régime se reflète dans la suppression possible de l'avocat, de certaines voies de recours et dans l'égalité des peines des 16-18 ans avec les adultes (y compris la peine capitale) (p. 133). Dans l'étude de Gérard Mauger sur les classes populaires entre 1975 et 2005, on trouve des éléments plus concrets sur les styles de vie déviants des jeunes selon trois catégories : les bandes, le milieu et la « bohème populaire » (p. 248).

11 La construction d'une identité juvénile féminine et l'approche de genre ne sont pas négligées dans Jeunesse oblige. Christine Bard met en avant le phénomène de la garçonne (une jeune femme) avec le succès du roman éponyme de Victor Margueritte (1922). Elle montre comment la face conservatrice du roman n'est pas perçue par les contemporains, obnubilés par la thèse avant-gardiste de la libération sexuelle des 
femmes (p. 113). Le personnage de la garçonne continue d'ailleurs d'incarner cette libération sexuelle dans les années 1960 (p. 126). Loin du caractère sulfureux de la garçonne, les travailleuses familiales de la fin des années 1940 et des années 1950 participent à la reconstruction de la France. Richard Ivan Jobs explique comment ce "service civil féminin » (p. 138) consiste à aider les mères de famille à surmonter les difficultés et à former les jeunes filles à leur futur travail de mère et d'épouse. À l'instar des cadres, les travailleuses familiales utilisent les techniques tayloristes et fordistes pour moderniser les foyers, mais sans bénéficier de la moindre ascension sociale (pp. 150-151). Prenant l'exemple de la sexualité, Michel Bozon s'interroge à son tour sur les dissymétries entre femmes et hommes : très marquées dans les années 1950, ont-elles disparu ou se sont-elles seulement déplacées dans les années 2000 (p. 227) ? Analysant les résultats d'enquêtes datant de 1950, 1970 (la célèbre enquête Simon) et de 2008 (qu'il a co-dirigée), il repère un rapprochement dans les âges d'entrée en sexualité des femmes et des hommes, une plus grande égalité dans les questions qui leur sont posées, mais revient sur le fait que les femmes sont toujours représentées de nos jours comme «agents civilisateurs des hommes » (p. 243).

12 Qui sont vraiment les jeunes ? Quel est leur rapport à l'histoire ? Telles sont les problématiques qui traversent tout l'ouvrage, mais qui sont particulièrement vives dans deux contributions. De la Libération à la fin de la guerre d'Algérie, les jeunes «grandissent dans la mémoire du conflit, sont élevés dans ses ombres et conviés à son souvenir » (p. 154) note Ludivine Bantigny. Les nombreuses enquêtes, livres et articles sur la jeunesse livrent une vision des jeunes garçons comme "sang neuf », comme facteurs de «redressement de l'histoire» durant la guerre d'Algérie, après la faillite des pères lors de la deuxième guerre mondiale. Mais la jeunesse a le «sentiment d'être consumée par la guerre » comme si elle n'avait pas d'histoire propre (p. 166). C'est aussi ce qui se passe pour ceux que Ivan Jablonkla appelle « les jeunes sans nom ». Avec les incidents des Minguettes en 1981, des jeunes qualifiés de « deuxième génération » font leur apparition sur la scène publique. Les pouvoirs publics les désigneront ensuite sous les termes de «jeunes issus de l'immigration » (p. 282), puis de «jeunes des cités » ou de «jeunes en situation d'exclusion» (pp. 286-287). Ces termes, apparemment neutres, désignent implicitement l'immigration maghrébine et africaine et se rapportent donc à une dimension ethnique et au passé colonial de la France qu'il s'agit d'occulter.

Mais qu'ils n'aient pas de nom ou qu'ils soient noyés sous une foule d'appellations, les jeunes font l'histoire et forment part de l'histoire. C'est bien la leçon magistrale de ce livre qui devrait inciter de jeunes historiens et historiennes à poursuivre les recherches sur la jeunesse. 